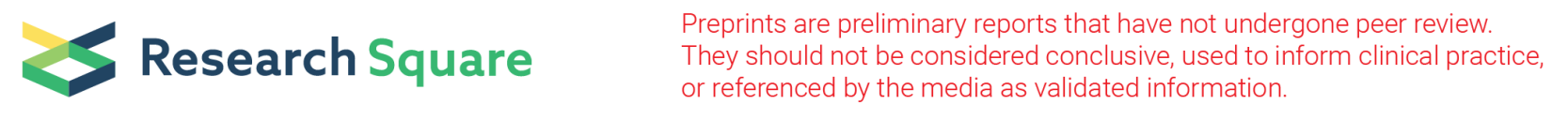

\title{
Predicted antiviral drugs Darunavir, Indinavir and Rimantadine can potentially bind to neutralize COVID-19 conserved proteins
}

\author{
Umesh Chandra Halder ( $\square$ umeshchandrahalder@gmail.com ) \\ Department of Zoology, Raniganj Girls' College, Searsole -Rajbari, Raniganj, Paschim Bardhaman -713358, West Bengal, India
}

\section{Research}

Keywords: COVID-19, Antiviral drugs, Drug binding 3D-motif, Darunavir, Indinavir, Rimantadine, Endoribonuclease, Main Protease, Replicase

Posted Date: April 13th, 2020

DOI: https://doi.org/10.21203/rs.3.rs-22247/v1

License: (c) (1) This work is licensed under a Creative Commons Attribution 4.0 International License. Read Full License

Version of Record: A version of this preprint was published at Journal of Biological Research-Thessaloniki on August 4th, 2021. See the published version at https://doi.org/10.1186/s40709-021-00149-2. 


\section{Abstract}

Background: Novel Coronavirus disease 2019 or COVID-19 has become a threat to human society due to fast spreading and increasing mortality. It uses vertebrate hosts and presently deploying humans. Life cycle and pathogenicity of COVID-19 have already been deciphered and possible drug target tests are on the way.

Results: The present study was aimed to analyze conserved Endoribonuclease, protease and replicase proteins of the virus as targets to all known drugs. A bioinformatics based web server Drug ReposeER predicted several drug binding motifs in these analyzed proteins. Results revealed that anti-viral Darunavir, Indinavir and Rimantadine were the most potent to have 3D-drug binding motifs on COVID-19 proteins.

Conclusions: Repurposing of the antiviral drugs Darunavir, Indinavir and Rimantadine to treat COVID-19 infected persons could be useful that can potentially prevent thousands of human mortality.

\section{Background}

COVID-19 has become a menace to the human kind that imposed epidemic condition. Great efforts are being carried out by the scientists to develop vaccine or a potent drug to curb human mortality. The virus has several types of vertebrate hosts including human and transmission occurs through direct contact or aerosols. It's a positive sense RNA virus with a genome of $\sim 30 \mathrm{~kb}(1,2)$. Like every animal virus their proteins hijack the cellular machineries to complete life cycle. These proteins are of great interest to the scientists to develop specific drug or vaccine against them. Search and trial for potential inhibitory drugs is on the way but they were proven ineffective to prevent patient death (3). The present work based on the fact that enzymes of any virus remain structurally and chemically conserver as they have to interact with human proteins and have to carry out same biochemical processes within cell. In the present study three structurally known enzymes of COVID-19, i.e., NSP15 endoribonuclease (PDB ID: 6VWW) (4), Main Protease (PDB ID: 6M03) (5), and NSP9 Replicase (PDB ID: 6W4B) (6) have been analyzed using Drug ReposeER web server program (http://27.126.156.175/drreposed/) (7) for their possible binding sites (8) to all drugs available in drug bank. Coronavirus endoribonuclease (NSP15/EndoU) is a hexameric protein that preferentially recognizes and cleaves RNA (1, 2, 9) and EndoU also evades host mediated viral double-stranded RNA recognition (10). Main protease ( $\mathrm{M}^{\text {pro }}$ ) or NSP5 is essential for viral replication (1, 2, 11) whereas NSP9 replicase is dimeric and involved in viral RNA synthesis $(1,2,12,13)$.

\section{Results And Discussion}

DrReposER predicted numerous potential 3D-motifs of both left $(\mathrm{L})$ and right $(\mathrm{R})$ superpositions for 6VWW, 6M03, 6W4B (Supplementary 1,

Supplementary 2, Supplementary 3). Known drugs that bind these motifs either bind human cellular, bacterial or viral proteins. From the hit results 16 anti-viral drugs were selected for their target specificities and effectiveness. The anti-viral drugs were presented in tabular formats (Table 1 3). Results showed that 21 unique potential 3D-motifs were predicted for (6VWW) Endoribonuclease (Table 1), 13 for (6M03) main Protease (Table 2) and 6 for (6W4B) Replicase (Table 3). The drugs were further analyzed and compared for their binding towards all the target proteins (Table 4). Results revealed that Darunavir (Ligand ID: 017), Indinavir (Ligand ID: MK1) and S-adenosylmethionine (SAM) have binding sites both on 6VWW and 6M03, whereas Ribavirine and Rimantadine (Ligand ID: RIM) have binding sites on 6M03 and 6W4B. Moreover, Tipranavir binding was predicted on all target proteins (Table 4). Other anti-viral drugs, i.e., Amprenavir, Atazanavir, Lopinavir, Saquinavir, Torimifene, Amphetamine, Nelfinavir, Nevirapine, Ritonavir and Grazoprevir had binding sites on any of the three analyzed proteins. From the results Darunavir, Indinavir and Rimantadine have been prioritized for their greater inhibitory binding to these proteins. Darunavir had 5 binding motifs (3LEU-23VAL-6VAL, 72ILE157GLY-156VAL, 73LEU-80ILE-86ILE, 300LEU-212ILE-253ILE, and 300LEU-296ILE-253ILE) on 6VWW and 2 binding motifs (109GLY-200ILE293PRO and 133ASN-195GLY-194ALA) on 6M03 (Table1,2,4, Fig 1,2). Indinavir had 3 binding motifs (122VAL-119PRO-80ILE, 173VAL-170GLY169ILE and 321VAL-344PRO-323ILE) on 6VWW and a single motif (106ILE-109GLY-200ILE) on 6M03 (Table1,2,4 Fig 1,2) whereas Rimantadine had 3 predicted binding sites (255ALA-254SER-251GLY, 255ALA-254SER-258GLY and 285ALA-284SER-283GLY) on 6M03 and 3 binding sites (111VAL-108ALA-106SER, 111VAL-109ALA-106SER and 109ALA-106SER-105GLY) on 6W4B (Table2,3,4, Fig 2,3). Again minimum Root Mean Square Deviation (RMSD) for 6VWW-Darunavir and 6M03-Darunavir binding was $0.77 \AA$ and $0.76 \AA$ and known interaction for binding involves maximum of 22 and 26 residues respectively (Table 1,2). RMSD and residue binding of Indinavir to $6 \mathrm{VWW}$ and $6 \mathrm{M} 03$ were $0.87 \AA ̊ 80.82 \AA$, and 21 \& 22 residues respectively (Table 1,2). Rimantadine accounts for $6 \mathrm{M} 03$ and 6W4B binding with RMSD of $0.88 \AA$ \& $0.90 \AA$ and $9 \& 10$ residues respectively (Table 2,3 ).

From the resulted Table 4, it is evident that SAM, Tipranavir and Ribavirin showed predicted binding sites for the targets, however, Darunavir, Indinavir and Rimantadine binding outnumber them. Again some target sites coincide with Darunavir, Indinavir or Rimantadine as can be seen for Saquinavir. Hence, Darunavir, Indinavir and Rimantadine could be the choice of drugs to treat COVID-19. Along with these SAM may be administered which is not a classical anti-viral drug and have implications to treat Liver problems. Darunavir (DRV- Drugbank ID: DB01264) and Indinavir (IDV- Drugbank ID: DB00224) are members of Protease-inhibitor family of antiviral drugs to ward off HIV, on the other hand Rimantadine (RIM- Drugbank ID: DB00478) is an Influenza virus inhibitor (14). These drugs are potent to inhibit their designated targets and results of this analysis also provide data that these can effectively bind COVID-19 targeted proteins to inhibit disease outcome and prevent human death. 


\section{Conclusion}

The findings strongly suggest that combination of antiviral drugs, Darunavir, Indinavir and Rimantadine can potentially inhibit COVID-19 enzymatic proteins. The targeted proteins are highly conserved and due to this reason inhibiting those can effectively inhibit the entire virus. Where no such drugs are proving ineffective to save thousands of lives, these predicted combinations of drugs must be clinically tested to cure patients. Practical implication of the findings can effectively save thousands of valuable lives. On the other hand vaccine development is on the way but the limitations of vaccine use is that it can not protect already infected patients showing disease outcomes and in those cases only effective drugs can save lives.

\section{Methods}

\section{Key Resources Table}

\begin{tabular}{|c|c|c|}
\hline Resource & Source & Identifier \\
\hline \multicolumn{3}{|l|}{ Deposited Data } \\
\hline COVI-19 Endonuclease 3D-structure & Kim et al., 2020 & PDB ID: 6VWW \\
\hline COVI-19 Main Protease 3D-structure & Zhang et al., 2020 & PDB ID: 6M03 \\
\hline COVI-19 Replicase 3D-structure & Tan et al., 2020 & PDB ID: 6W4B \\
\hline \multicolumn{3}{|l|}{ Web Server } \\
\hline DrReposER & Ab Ghani et al., 2019 & http://27.126.156.175/drreposed/ \\
\hline
\end{tabular}

DrReposER has been used to find binding interfaces or 3D-motifs of target proteins (PDB ID: 6VWW, 6M03, 6W4B) for all possible drugs. The program uses SPRITE and ASSAM web servers to find amino acid side chains $(8,15,16,17)$. Drug ReposER compares structurally similar side chain arrangements from PDB repository and assign hit results for different drug targets in the query PDB ID.

\section{Declarations}

Ethics approval and consent to participate: Not applicable

Consent for publication : Not applicable

Availability of data and materials : Not applicable

Competing interests : The author has no competing interests

Funding : Not applicable

Authors' contributions : Dr. Umesh Chandra Halder has designed, performed all analysis, written the paper, and prepared the images and Tables.

Acknowledgements : Not applicable

\section{References}

1. Chen Y, Liu Q, Guo D. Emerging coronaviruses: Genome structure, replication, and pathogenesis. J Med Virol. 2020;92:418-423.

2. Fehr AR, Perlman S. Coronaviruses: an overview of their replication and pathogenesis. Methods Mol Biol.2015;1282: 1-23.

3. Dong L, Hu S, Gao J. Discovering drugs to treat coronavirus disease 2019 (COVID-19). Drug Discov Ther.2020;14:58-60.

4. Kim Y, Jedrzejczak R, Maltseva N, Endres M, Godzik A, Michalska K, et al. Crystal Structure of NSP15 Endoribonuclease from SARS CoV-2. PDB data. 2020. https://www.rcsb.org/structure/6VWW.

5. Zhang B, Zhao Y, Jin Z, Liu X, Yang H, Rao Z. The crystal structure of COVID-19 main protease in apo form. PDB data. 2020. http://www.rcsb.org/structure/6M03.

6. Tan K, Kim Y, Jedrzejczak R, Maltseva N, Endres M, Michalska K, et al. The crystal structure of Nsp9 RNA binding protein of SARS CoV-2. PDB data. $2020 \mathrm{http}: / /$ www.rcsb.org/structure/6W4B.

7. Drug ReposeER web server program. http://27.126.156.175/drreposed/. Accessed 25 March 2020.

8. Ab Ghani NS, Ramlan El, Firdaus-Raih M. Drug ReposER: a web server for predicting similar amino acid arrangements to known drug binding interfaces for potential drug repositioning. Nucleic acids research. 2019;47:W350-W356. 
9. Bhardwaj K, Sun J, Holzenburg A, Guarino LA, Kao CC. RNA recognition and cleavage by the SARS coronavirus endoribonuclease. J Mol Biol. 2006;361:243-56.

10. Deng X, Baker SC. An "Old" protein with a new story: Coronavirus endoribonuclease is important for evading host antiviral defenses. Virology. 2018;517:157-163.

11. Wang F, Chen C, Tan W, Yang K, Yang H. Structure of Main Protease from Human Coronavirus NL63: Insights for Wide Spectrum AntiCoronavirus Drug Design. Sci Rep. 2016;6:22677.

12. Sutton G, Fry E, Carter L, Sainsbury S, Walter T, Nettleship J, et al. The nsp9 replicase protein of SARS-coronavirus, structure and functional insights. Structure. 2004;12:341-53.

13. Ziebuhr J. The coronavirus replicase. Curr Top Microbiol Immunol. 2005;287:57-94.

14. De Clercq E, Li G. Approved Antiviral Drugs over the Past 50 Years. Clin Microbiol Rev. 2016;29:695-747.

15. Nadzirin N, Gardiner E, Willett P, Artymiuk PJ, Firdaus-Raih M. SPRITE and ASSAM: web servers for side chain 3D-motif searching in protein structures. Nucleic Acids Res.2012;40:W380-6.

16. Spriggs RV, Artymiuk PJ, Willett P. Searching for patterns of amino acids in 3D protein structures. J Chem Inf Comput Sci. 2003;43:412-21.

17. Artymiuk PJ, Poirrette AR, Grindley HM, Rice DW, Willett P. A graph-theoretic approach to the identification of three-dimensional patterns of amino acid side-chains in protein structures.J Mol Biol. 1994;243:327-44.

\section{Tables}


Table 1. Possible binding sites of NSP15 against known anti-viral drugs. Features of different drug binding motifs. HIV-1-Human Immunodeficiency virus 1, RMSD- Root Mean Square Deviation, A-angstrom, ILE-isoleucine, GLY-glycine, VAL-valine, LEUleucine, PRO-proline, ASP-aspartic acid

\begin{tabular}{|c|c|c|c|c|c|}
\hline & & & & & $\begin{array}{lr}\text { (6VWW) } & \text { NSP15 } \\
\text { ENDORIBONUCLEASE OF COVID-19 }\end{array}$ \\
\hline DRUGS & & $\begin{array}{l}\text { TOTAL } \\
\text { BINDING } \\
\text { SITES }\end{array}$ & & & \\
\hline \multirow[t]{11}{*}{ AMPRENAVIR } & \multicolumn{4}{|c|}{ KNOWN SIMILAR TARGET MOLECULE } & PROTEASE, HIV-1 \\
\hline & \multirow{10}{*}{$\begin{array}{l}\text { BINDING } \\
\text { PROPERTIES }\end{array}$} & \multirow[t]{10}{*}{2} & \multirow[t]{5}{*}{1} & SUPERPOSITION TYPE & $\mathrm{L}$ \\
\hline & & & & RMSD & $0.90 \AA$ \\
\hline & & & & $\begin{array}{l}\text { AMINO ACID TARGETS } \\
\text { OF DRUG }\end{array}$ & $\begin{array}{l}72 \text { ILE } \\
157 \text { GLY } \\
156 \text { VAL }\end{array}$ \\
\hline & & & & $\begin{array}{lcc}\text { NO. OF } & \text { RESIDUES } & \text { IN } \\
\text { KNOWN BINDING } & \end{array}$ & 25 \\
\hline & & & & $\begin{array}{ll}\text { HUMAN } & \text { SIMILAR } \\
\text { TARGETS } & \end{array}$ & 4 \\
\hline & & & \multirow[t]{5}{*}{2} & SUPERPOSITION TYPE & $\mathrm{L}$ \\
\hline & & & & RMSD & $0.66 \AA$ \\
\hline & & & & $\begin{array}{l}\text { AMINO ACID TARGETS } \\
\text { OF DRUG }\end{array}$ & $\begin{array}{l}251 \text { LEU } \\
276 \text { VAL } \\
296 \text { ILE }\end{array}$ \\
\hline & & & & $\begin{array}{l}\text { NO. OF } \text { RESIDUES } \\
\text { KNOWN BINDING }\end{array}$ & 19 \\
\hline & & & & $\begin{array}{ll}\text { HUMAN } & \text { SIMILAR } \\
\text { TARGETS } & \end{array}$ & 5 \\
\hline
\end{tabular}

KNOWN SIMILAR TARGET MOLECULE BINDING
PROPERTIES

KNOWN SIMILAR TARGET MOLECULE

BINDING

PROPERTIES

\begin{tabular}{|l|l|}
\hline RUPERPOSITION TYPE & \\
\hline AMINO ACID TARGETS & \\
OF DRUG & \\
\hline $\begin{array}{l}\text { NO. OF RESIDUES IN } \\
\text { KNOWN BINDING }\end{array}$ & $\begin{array}{l}\text { HUMAN SIMILAR } \\
\text { TARGETS }\end{array}$ \\
\hline
\end{tabular}

PROTEASE, HIV-1

$0.83 \AA$

72 ILE

157 GLY

156 VAL

24

4

\section{DARUNAVIR}




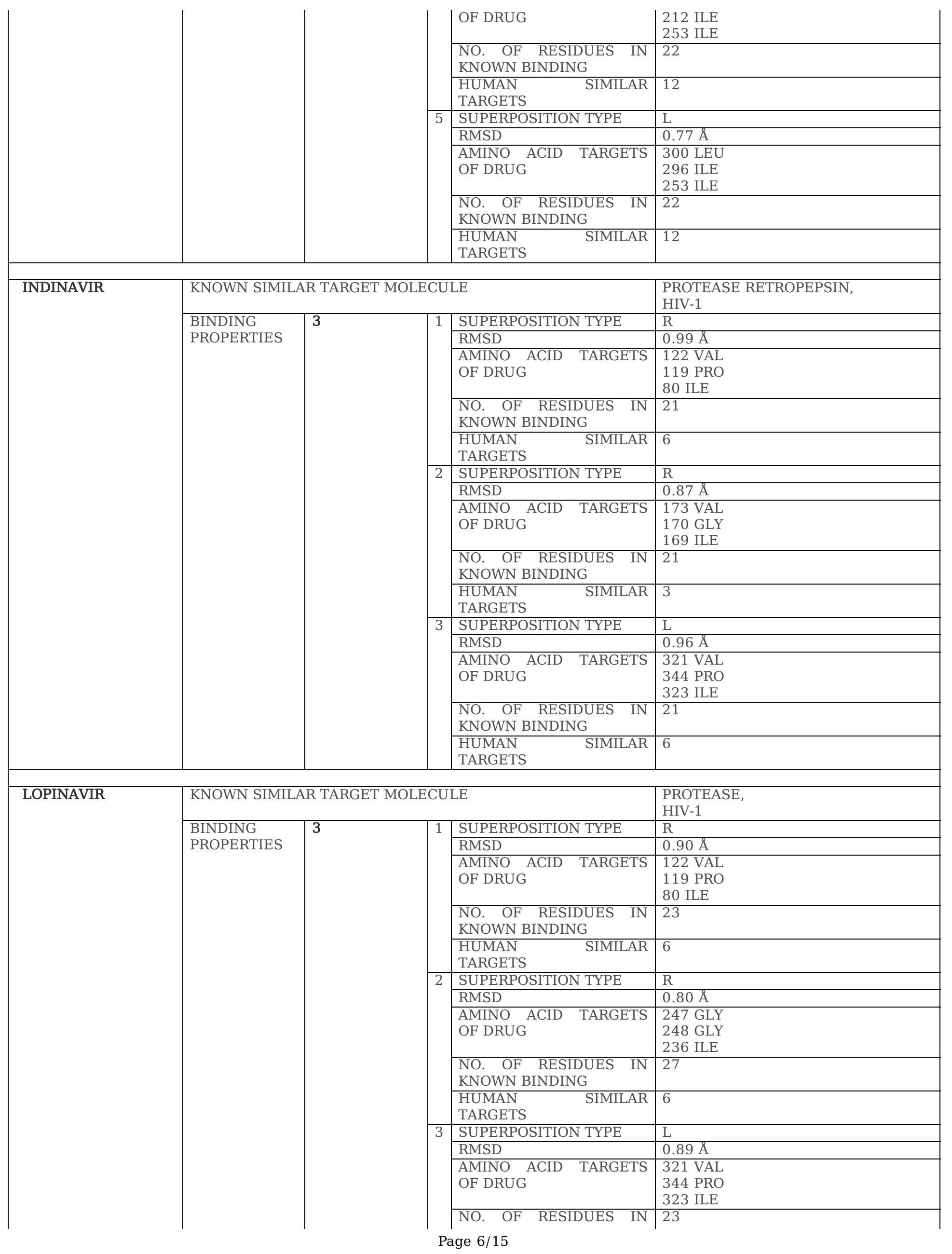




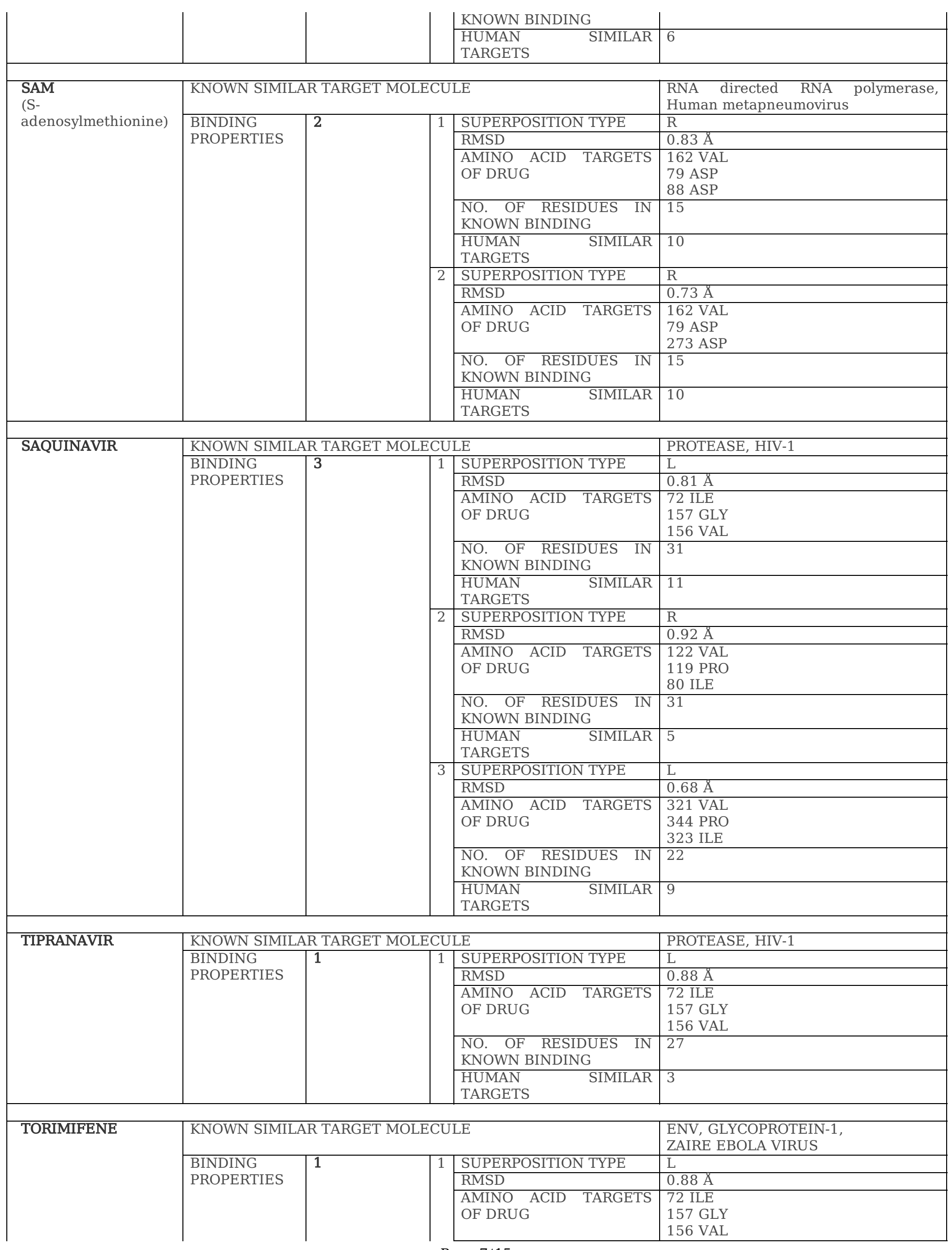




\begin{tabular}{|l|l|l|l|l|} 
& & $\begin{array}{l}\text { NO. OF RESIDUES IN } \\
\text { KNOWN BINDING 27 } \\
\text { HUMAN SIMILAR } \\
\text { TARGETS }\end{array}$ \\
\hline
\end{tabular}

Page 8/15 
Table 2. Possible binding sites of Protease against known anti-viral drugs. Features of different drug binding motifs. HIV-1Human Immunodeficiency virus 1, RMSD- Root Mean Square Deviation, §-angstrom, ILE-isoleucine, GLY-glycine, VAL-valine, LEU-leucine, PRO-proline, ASP-aspartic acid, ASN-asparagine, ALA-alanine, THR-threonine, LYS-lysine, SER-serine.

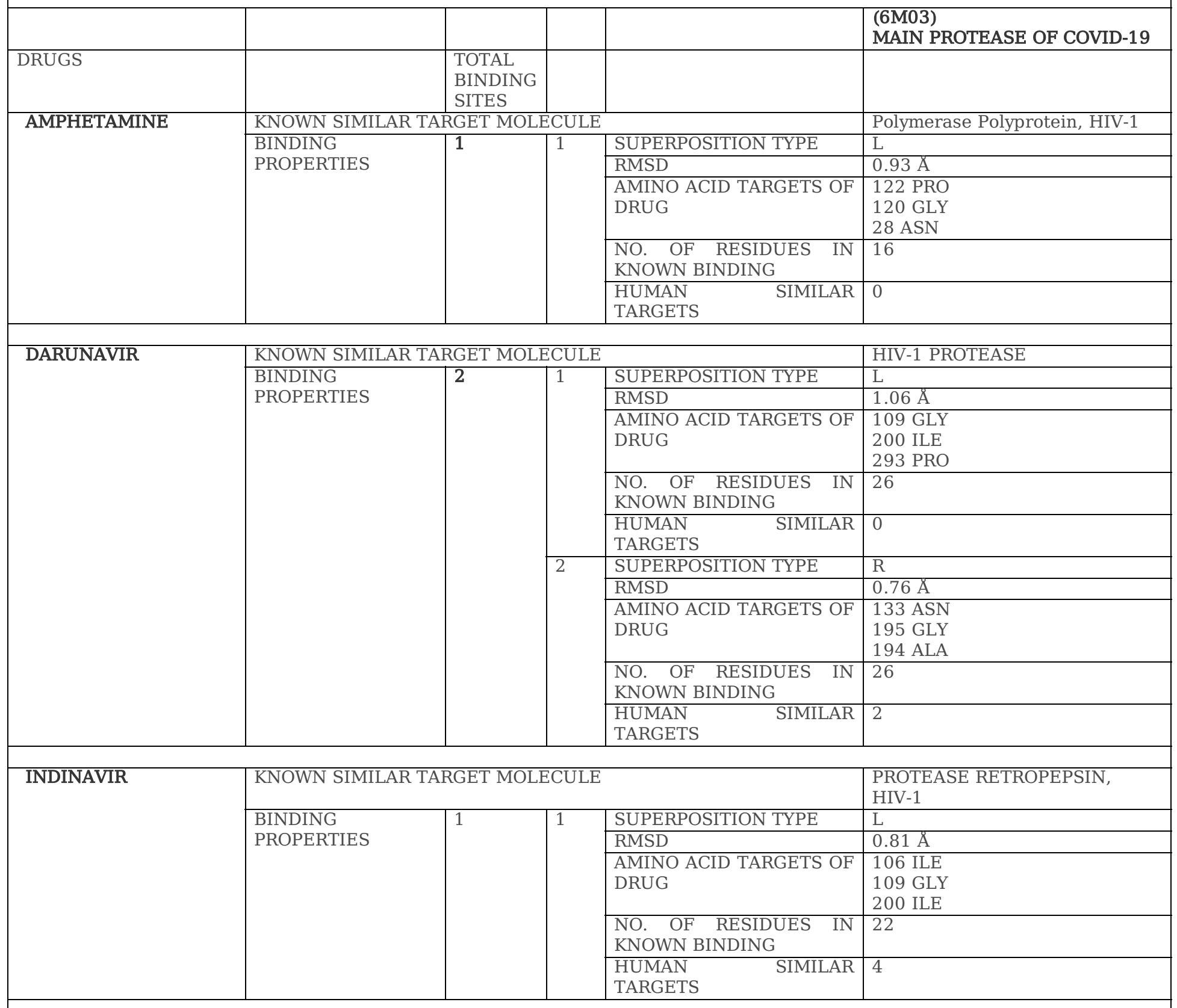

\begin{tabular}{|c|c|c|c|c|c|}
\hline \multirow[t]{7}{*}{ NELFINAVIR } & \multicolumn{4}{|c|}{ KNOWN SIMILAR TARGET MOLECULE } & PROTEASE RETROPEPSIN, \\
\hline & \multirow{6}{*}{$\begin{array}{l}\text { BINDING } \\
\text { PROPERTIES }\end{array}$} & \multirow[t]{6}{*}{1} & \multirow[t]{6}{*}{1} & SUPERPOSITION TYPE & $\mathrm{L}$ \\
\hline & & & & RMSD & $1.05 \AA$ \\
\hline & & & & AMINO ACID TARGETS OF & 153 ASP \\
\hline & & & & DRUG & $\begin{array}{l}292 \text { THR } \\
293 \text { PRO }\end{array}$ \\
\hline & & & & $\begin{array}{l}\text { NO. OF RESIDUES } \\
\text { KNOWN BINDING }\end{array}$ & 30 \\
\hline & & & & $\begin{array}{l}\text { HUMAN SIMILAR } \\
\text { TARGETS }\end{array}$ & 13 \\
\hline \multirow[t]{5}{*}{ NEVIRAPINE } & \multicolumn{4}{|c|}{ KNOWN SIMILAR TARGET MOLECULE } & $\begin{array}{l}\text { REVERSE TRANSCRIPTASE, } \\
\text { HIV-1 }\end{array}$ \\
\hline & \multirow{4}{*}{$\begin{array}{l}\text { BINDING } \\
\text { PROPERTIES }\end{array}$} & \multirow[t]{4}{*}{1} & \multirow[t]{4}{*}{1} & SUPERPOSITION TYPE & $\mathrm{R}$ \\
\hline & & & & RMSD & $1.10 \AA$ \\
\hline & & & & $\begin{array}{l}\text { AMINO ACID TARGETS OF } \\
\text { DRUG }\end{array}$ & $\begin{array}{l}88 \text { LYS } \\
\text { 86 VAL }\end{array}$ \\
\hline & & & & & $30 \mathrm{LEU}$ \\
\hline
\end{tabular}




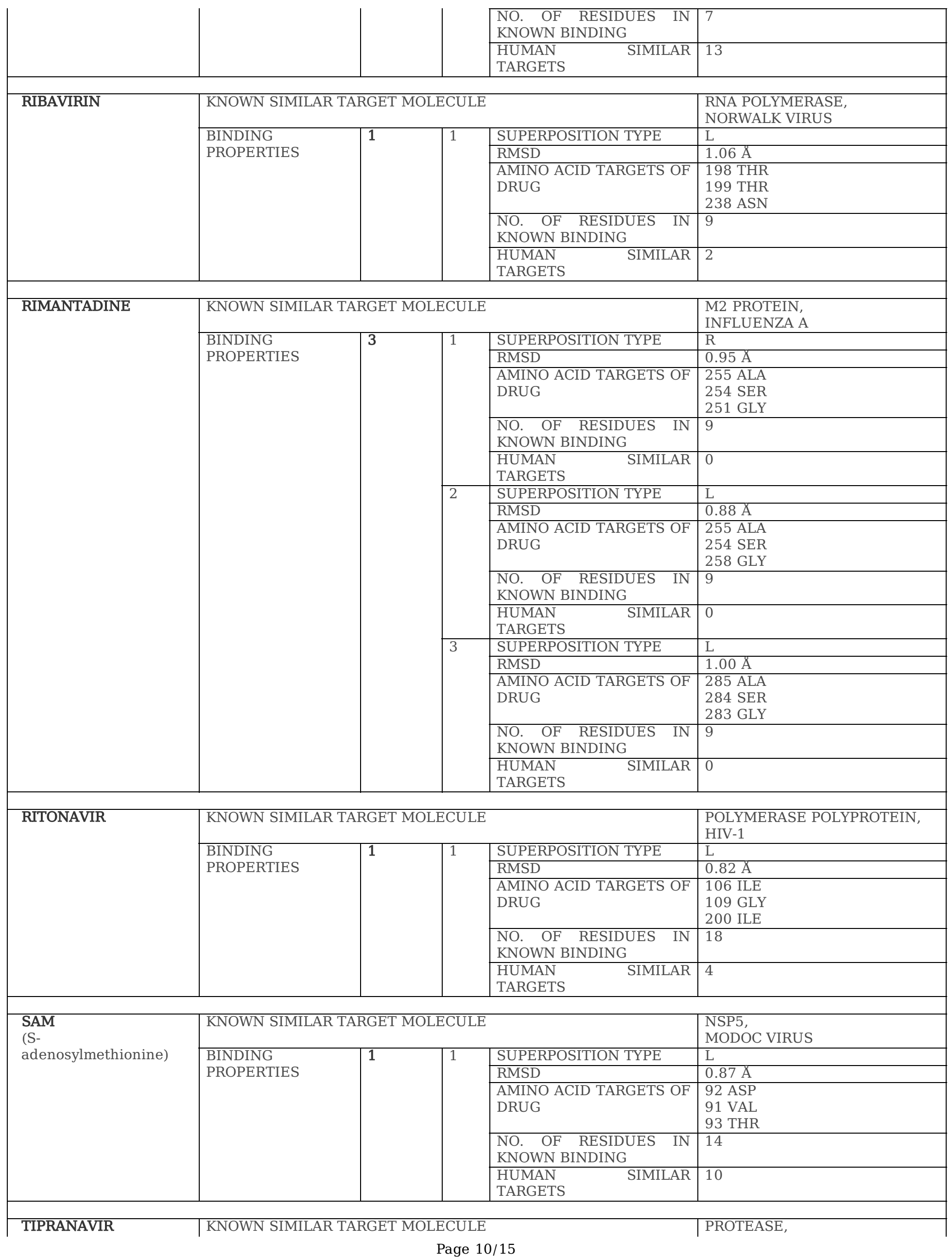




\begin{tabular}{|c|c|c|c|c|}
\hline & & & & HIV-1 \\
\hline \multirow{7}{*}{$\begin{array}{l}\text { BINDING } \\
\text { PROPERTIES }\end{array}$} & \multirow[t]{7}{*}{1} & \multirow[t]{7}{*}{1} & SUPERPOSITION TYPE & $\mathrm{L}$ \\
\hline & & & RMSD & $1.17 \AA$ \\
\hline & & & AMINO ACID TARGETS OF & 94 ALA \\
\hline & & & DRUG & 34 ASP \\
\hline & & & & 33 ASP \\
\hline & & & $\begin{array}{lcc}\text { NO. OF } & \text { RESIDUES } & \text { IN } \\
\text { KNOWN BINDING } & \end{array}$ & 27 \\
\hline & & & $\begin{array}{ll}\text { HUMAN } & \text { SIMILAR } \\
\text { TARGETS } & \end{array}$ & 3 \\
\hline
\end{tabular}

Table 3. Possible binding sites of NSP9 against known anti-viral drugs. Features of different drug binding motifs. HIV-1-Human Immunodeficiency virus 1, RMSD- Root Mean Square Deviation, A-angstrom, ILEisoleucine, GLY-glycine, VAL-valine, ASP-aspartic acid, ASN-asparagine, ALA-alanine, THR-threonine, LYSlysine, SER-serine.

\begin{tabular}{|c|c|c|c|c|c|}
\hline & & & & & $\begin{array}{l}\text { (6W4B) } \\
\text { NSP9 Replicase of } \\
\text { COVID-19 }\end{array}$ \\
\hline DRUGS & & $\begin{array}{ll}\text { TOTAL } & \text { BINDING } \\
\text { SITES } & \end{array}$ & & & \\
\hline \multirow[t]{6}{*}{ GRAZOPREVIR } & \multicolumn{4}{|c|}{ KNOWN SIMILAR TARGET MOLECULE } & $\begin{array}{l}\text { NS3 PROTEASE, } \\
\text { PROTEIN, } \\
\text { Hepacivirus C } \\
\end{array}$ \\
\hline & \multirow[t]{5}{*}{$\begin{array}{l}\text { BINDING } \\
\text { PROPERTIES }\end{array}$} & \multirow[t]{5}{*}{1} & \multirow[t]{5}{*}{1} & $\begin{array}{l}\text { SUPERPOSITION } \\
\text { TYPE }\end{array}$ & $\mathrm{L}$ \\
\hline & & & & RMSD & $0.94 \AA$ \\
\hline & & & & $\begin{array}{lr}\text { AMINO } & \text { ACID } \\
\text { TARGETS } & \text { OF } \\
\text { DRUG } & \end{array}$ & $\begin{array}{l}66 \text { ILE } \\
59 \text { LYS } \\
62 \text { GLY }\end{array}$ \\
\hline & & & & $\begin{array}{ll}\text { NO. } & \text { OF } \\
\text { RESIDUES } & \text { IN } \\
\text { KNOWN } & \\
\text { BINDING } & \end{array}$ & 16 \\
\hline & & & & $\begin{array}{l}\text { HUMAN } \\
\text { SIMILAR } \\
\text { TARGETS }\end{array}$ & 8 \\
\hline
\end{tabular}

\begin{tabular}{|c|c|c|c|c|c|}
\hline \multirow[t]{6}{*}{ RIBAVIRIN } & \multicolumn{4}{|c|}{ KNOWN SIMILAR TARGET MOLECULE } & RNA POLYMERASE, \\
\hline & \multirow[t]{5}{*}{$\begin{array}{l}\text { BINDING } \\
\text { PROPERTIES }\end{array}$} & \multirow[t]{5}{*}{1} & \multirow[t]{5}{*}{1} & $\begin{array}{l}\text { SUPERPOSITION } \\
\text { TYPE }\end{array}$ & $\mathrm{R}$ \\
\hline & & & & RMSD & $0.80 \AA$ \\
\hline & & & & $\begin{array}{lr}\text { AMINO } & \text { ACID } \\
\text { TARGETS } & \text { OF } \\
\text { DRUG } & \end{array}$ & $\begin{array}{l}36 \text { THR } \\
35 \text { THR } \\
34 \text { ASN }\end{array}$ \\
\hline & & & & $\begin{array}{ll}\text { NO. } & \text { OF } \\
\text { RESIDUES } & \text { IN } \\
\text { KNOWN } & \\
\text { BINDING } & \\
\end{array}$ & 09 \\
\hline & & & & $\begin{array}{l}\text { HUMAN } \\
\text { SIMILAR } \\
\text { TARGETS }\end{array}$ & 2 \\
\hline
\end{tabular}

\begin{tabular}{|c|c|c|c|c|c|}
\hline \multirow[t]{7}{*}{ RIMANTADINE } & \multicolumn{4}{|c|}{ KNOWN SIMILAR TARGET MOLECULE } & $\begin{array}{l}\text { M2, BM2 PROTEIN, } \\
\text { INFLUENZA A,B }\end{array}$ \\
\hline & \multirow[t]{6}{*}{$\begin{array}{l}\text { BINDING } \\
\text { PROPERTIES }\end{array}$} & \multirow[t]{6}{*}{3} & \multirow[t]{5}{*}{1} & $\begin{array}{l}\text { SUPERPOSITION } \\
\text { TYPE }\end{array}$ & $\mathrm{L}$ \\
\hline & & & & RMSD & $0.90 \AA$ \\
\hline & & & & $\begin{array}{lr}\text { AMINO } & \text { ACID } \\
\text { TARGETS } & \text { OF } \\
\text { DRUG } & \end{array}$ & $\begin{array}{l}109 \text { ALA } \\
106 \text { SER } \\
105 \text { GLY }\end{array}$ \\
\hline & & & & $\begin{array}{lr}\text { NO. } & \text { OF } \\
\text { RESIDUES } & \text { IN } \\
\text { KNOWN } & \\
\text { BINDING } & \end{array}$ & 9 \\
\hline & & & & $\begin{array}{l}\text { HUMAN } \\
\text { SIMILAR } \\
\text { TARGETS }\end{array}$ & 0 \\
\hline & & & 2 & SUPERPOSITION & $\mathrm{R}$ \\
\hline
\end{tabular}

Page $11 / 15$ 


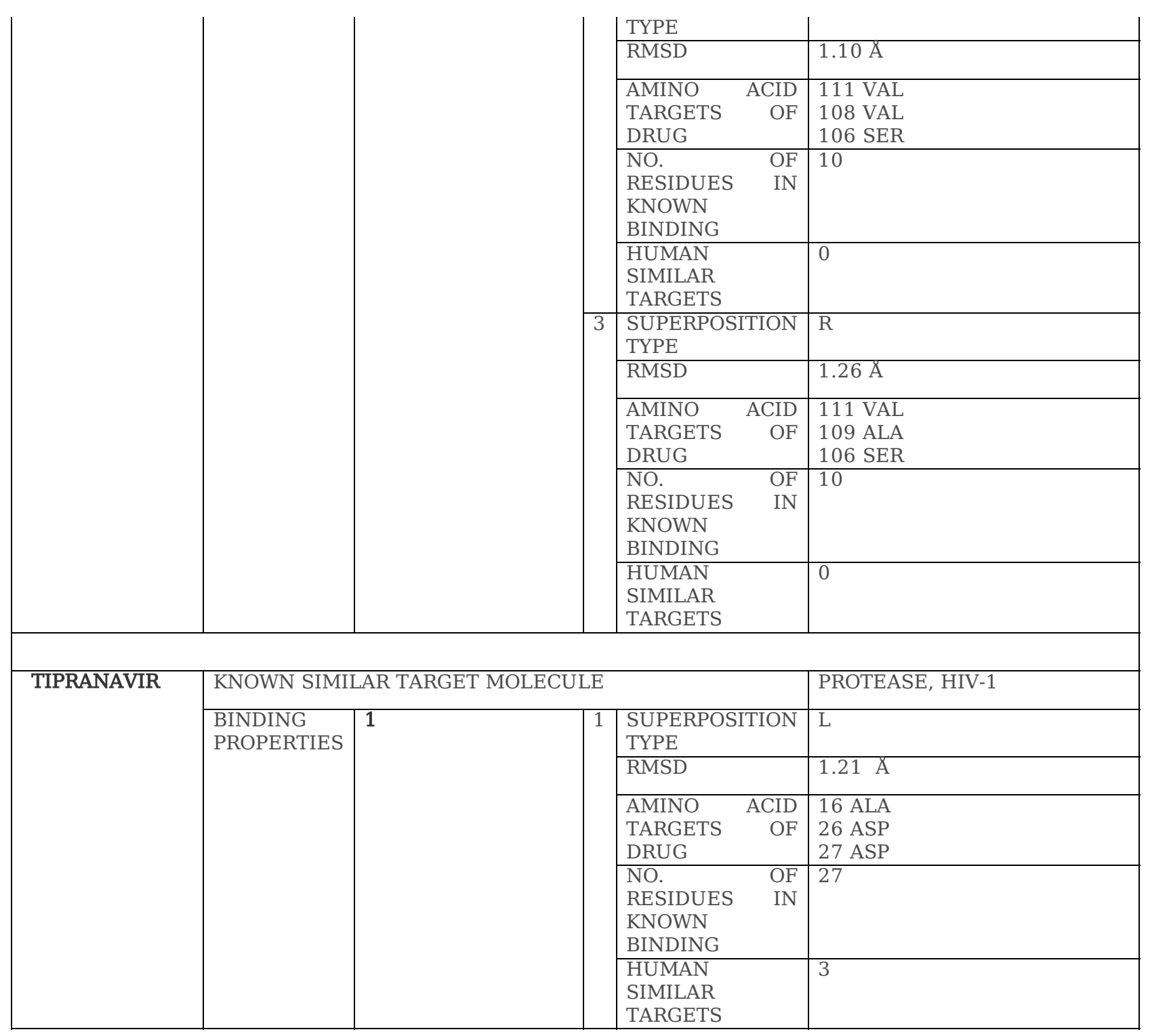

Table 4. Comparison of binding sites of all selected drugs. Out of 16 anti-viral drugs 3 have been selected for their greater binding.

\begin{tabular}{|c|c|c|c|}
\hline DRUGS & 6VWW & 6M03 & 6W4B \\
\hline Amphetamine & - & + & - \\
\hline Amprenavir & ++ & - & - \\
\hline Atazanavir & + & - & - \\
\hline Darunavir & +++++ & ++ & - \\
\hline Grazoprevir & - & - & + \\
\hline Indinavir & +++ & + & - \\
\hline Lopinavir & +++ & - & - \\
\hline Nelfinavir & - & + & - \\
\hline Nevirapine & - & + & - \\
\hline Ribavirin & - & + & + \\
\hline Rimantadine & - & +++ & +++ \\
\hline Ritonavir & - & + & - \\
\hline S-adenosylmethionine & ++ & + & - \\
\hline Tipranavir & + & + & + \\
\hline Torimifene & + & - & - \\
\hline
\end{tabular}

\section{Figures}




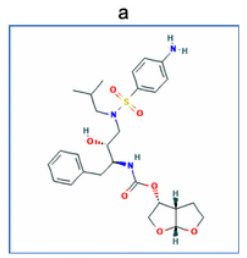

Darunavir

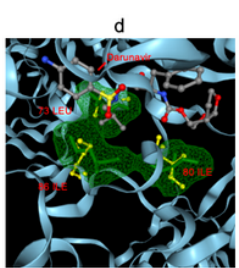

73-80-86

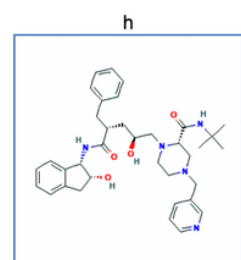

Indinavir

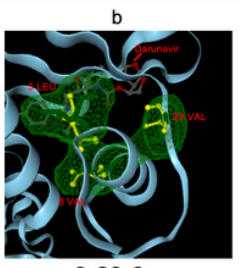

3-23-6

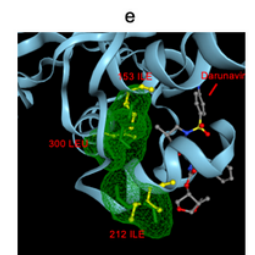

300-212-253

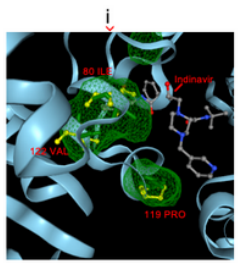

122-119-80

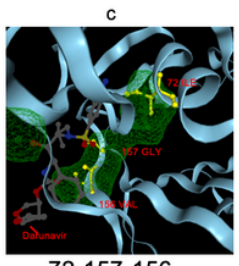

72-157-156

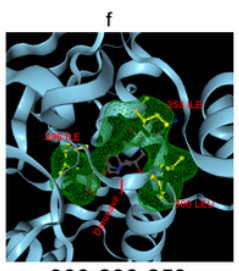

300-296-253

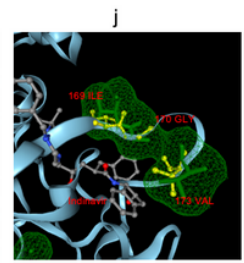

173-170-169
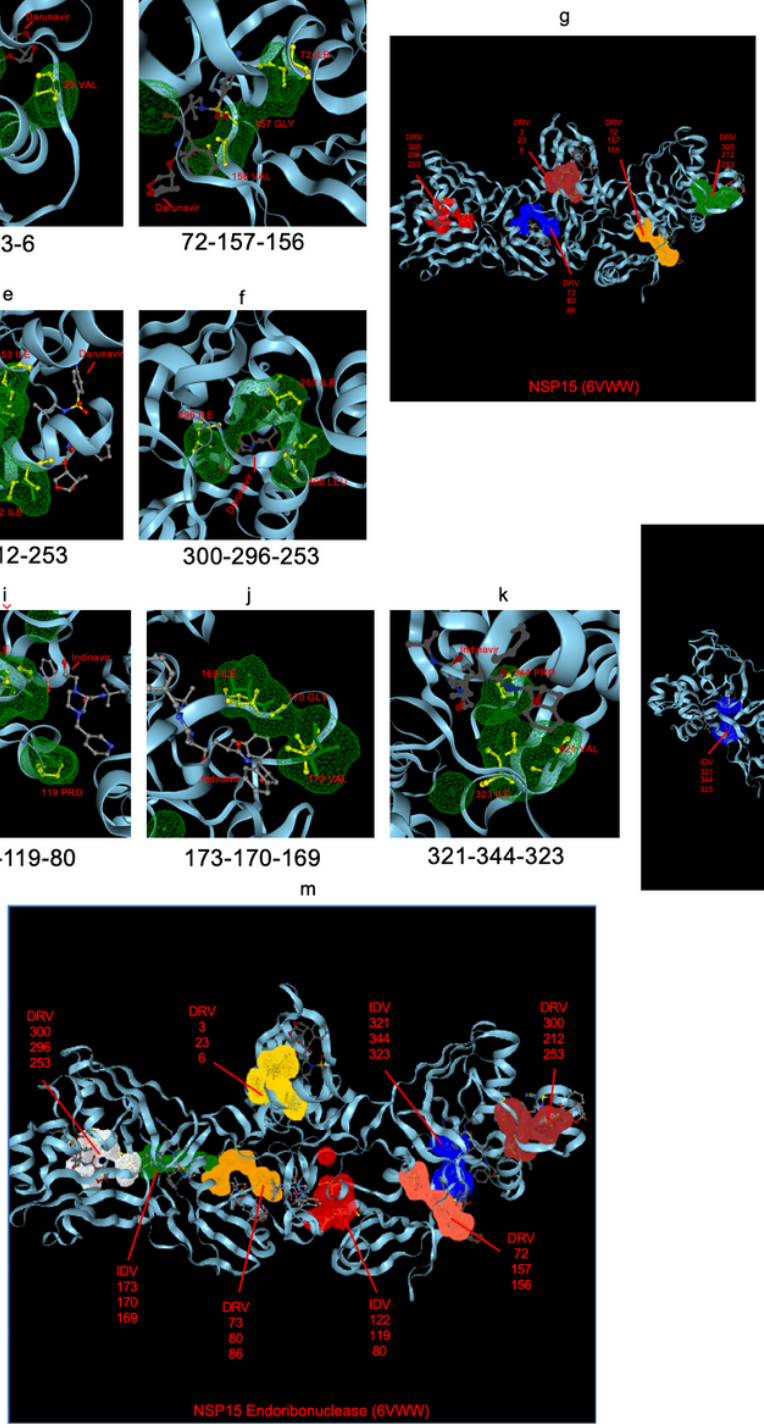
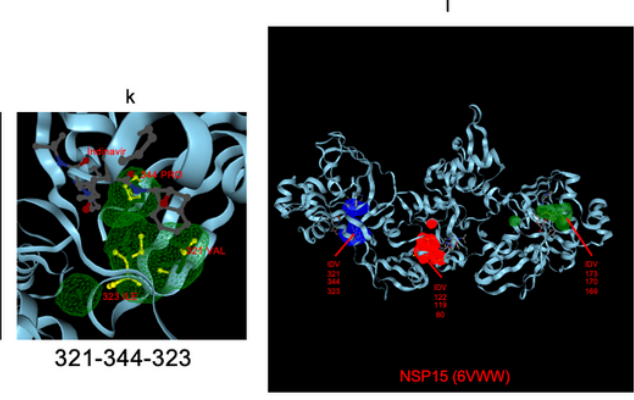

321-344-323

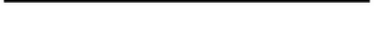

\section{Figure 1}

3D-binding interfaces of NSP15 Endoribonuclease with Darunavir \& Indinavir. a. Chemical structure of Darunavir. b-f. Binding pockets of Darunavir with different interacting motifs. g. All the binding pockets of NSP15 with Darunavir. h. Chemical structure of Indinavir. i-k. Binding pockets of Indinavir with different interacting motifs. I. All the binding pockets of NSP15 with Indinavir. m. Combined binding interfaces of Darunavir and Indinavir with NSP15. DRV-Darunavir, IDV-Indinavir. 
a

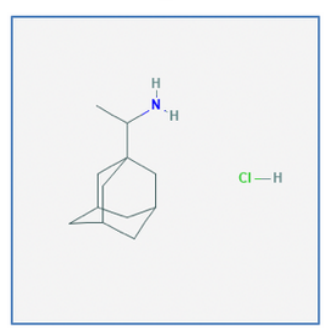

Rimantadine

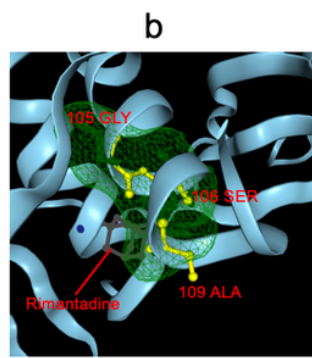

109-106-105

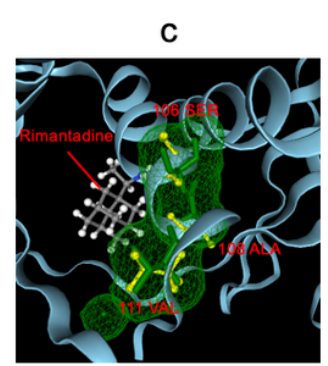

111-108-106

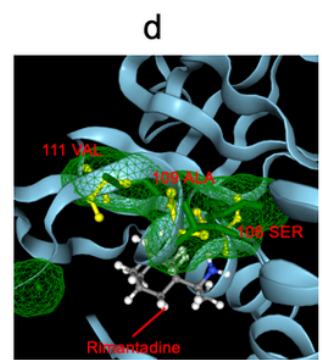

111-109-106

e

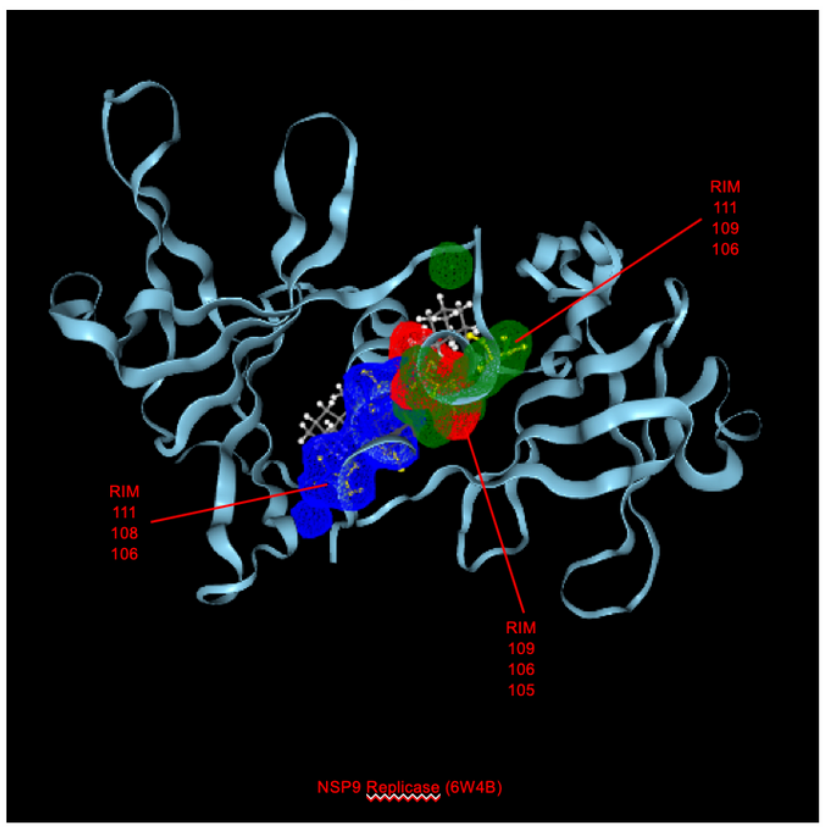

Figure 2

3D-binding interfaces of Main Protease with Rimantadine, Darunavir \& Indinavir. a. Chemical structure of Rimantadine. b-d. Binding pockets of Rimantadine with different interacting motifs. e. All the binding pockets of Protease with Rimantadine. f. Chemical structure of Darunavir. $\mathrm{g}$, $\mathrm{h}$. Binding pockets of Darunavir with different interacting motifs. i. All the binding pockets of Protease with Darunavir. j. Chemical structure of Indinavir. k. Binding pockets of Indinavir with different interacting motifs. I. Combined binding interfaces of Rimantadine, Darunavir and Indinavir with Main Protease. RIM-Rimantadine, DRV-Darunavir, IDV-Indinavir. 


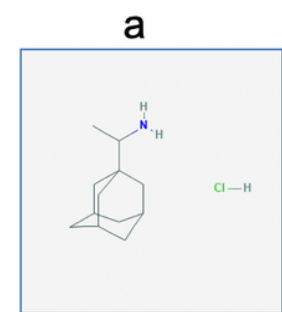

Rimantadine b

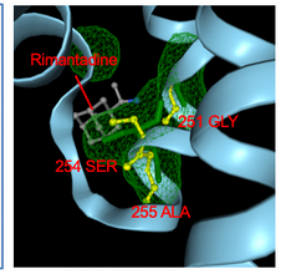

255-254-251
C

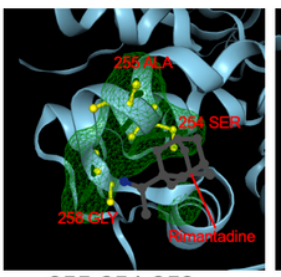

255-254-258 d

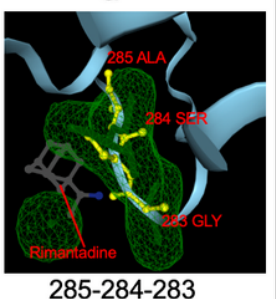

285-284-283
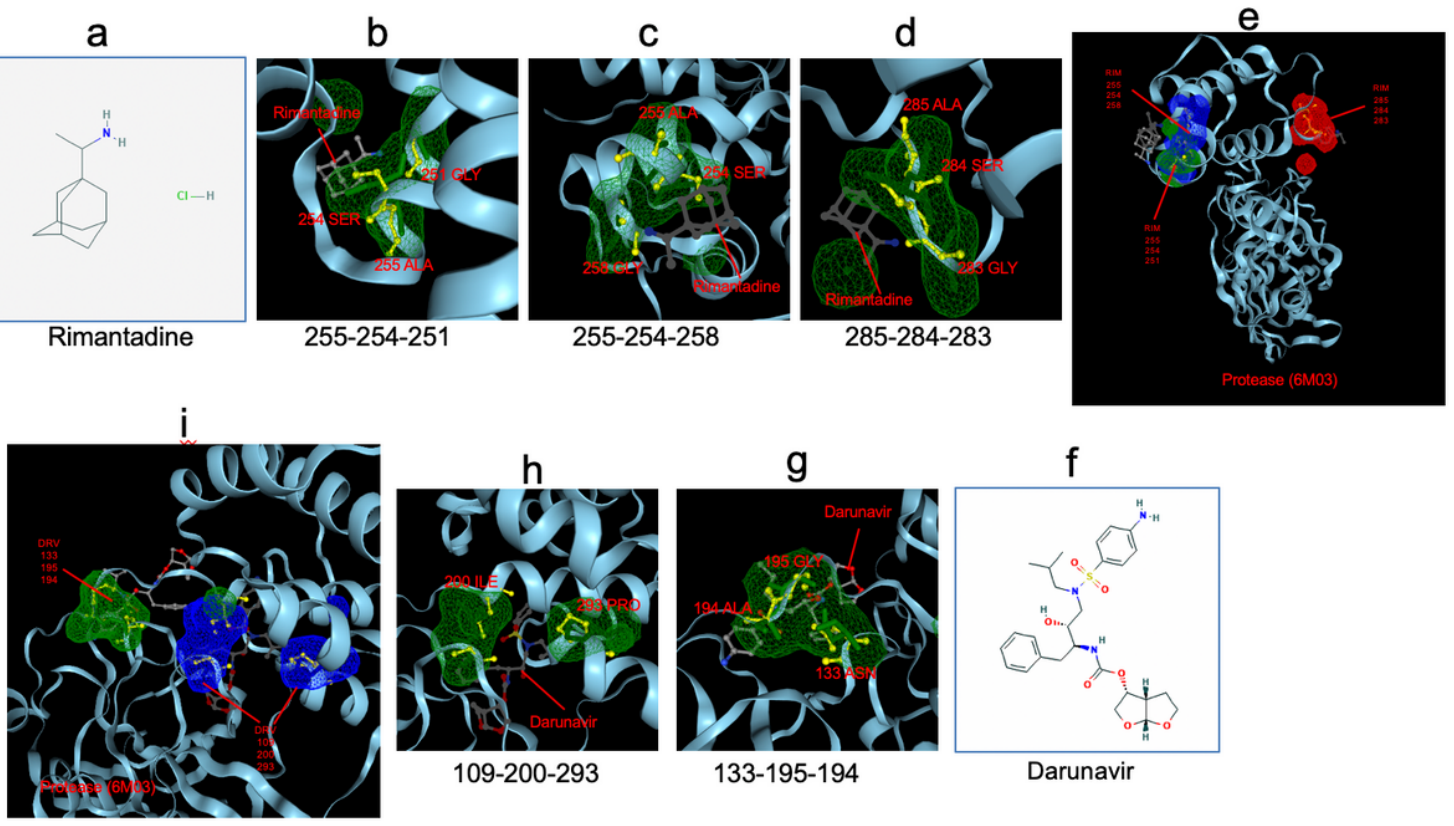

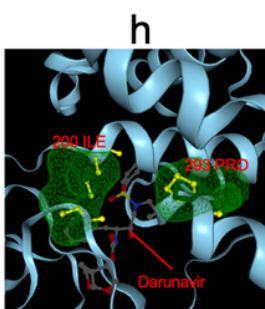

109-200-293

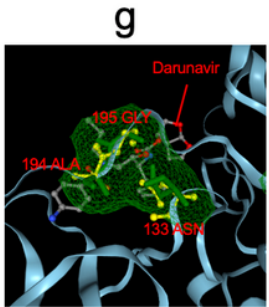

133-195-194

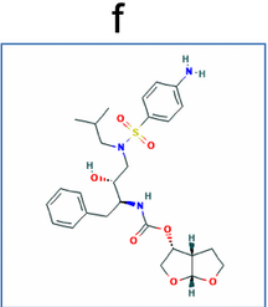

Darunavir

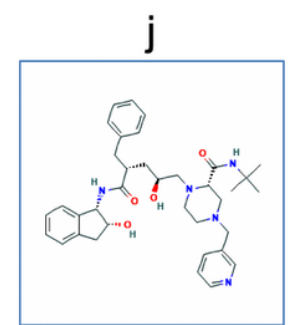

Indinavir

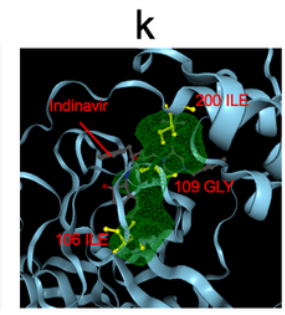

106-109-200

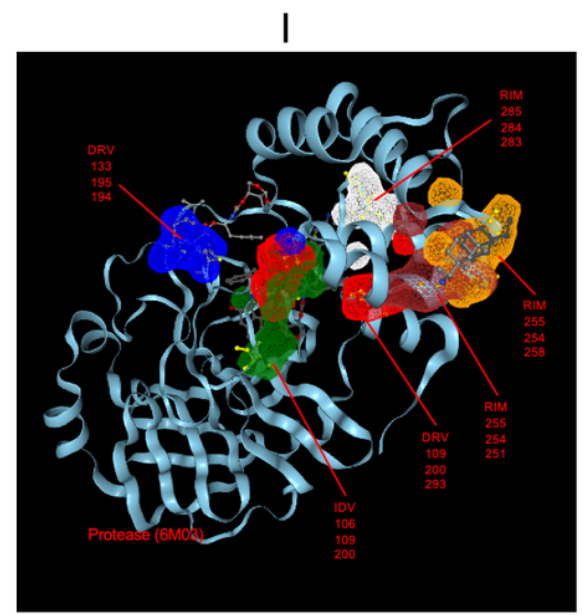

Figure 3

3D-binding interfaces of NSP9 Replicase with Rimantadine. a. Chemical structure of Rimantadine. b-d. Binding pockets of Rimantadine with different interacting motifs. e. All the binding pockets of Replicase with Rimantadine. RIM-Rimantadine.

\section{Supplementary Files}

This is a list of supplementary files associated with this preprint. Click to download.

- S1LISTOFHITSFOR6VWW.docx

- S2LISTOFHITSOF6M03MAINPROTEASE.docx

- S3LISTOFHITSFOR6W4B.docx 